\title{
Early specialized care after a first unprovoked epileptic seizure
}

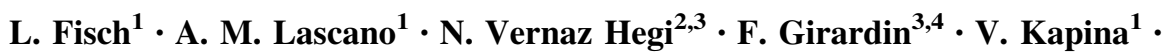

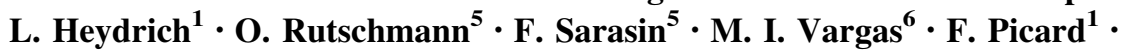 \\ S. Vulliémoz ${ }^{1}$ A. C. Héritier-Barras ${ }^{1}$ M. Seeck ${ }^{1}$
}

Received: 16 June 2016/Revised: 11 August 2016/Accepted: 12 August 2016/Published online: 7 September 2016

(c) Springer-Verlag Berlin Heidelberg 2016

\begin{abstract}
A first seizure is a life-changing event with physical and psychological consequences. We aimed to assess the role of early comprehensive patient care after a first unprovoked seizure to improve diagnostic accuracy and follow-up adherence. From April 2011 to March 2012, patients presenting a first unprovoked epileptic seizure received standard patient care (SPC), i.e., a consultation in the ED, an EEG and a CT scan. The patients were notified of the follow-ups. We compared this protocol to subsequently acquired "early comprehensive patient care" (ECPC), which included a consultation by an epileptologist in the emergency department (ED), a routine or long-term monitoring electroencephalogram (LTM-EEG), magnetic resonance imaging and three follow-up consultations (3 weeks, 3 months, 12 months). 183 patients were included (113 ECPC, 70 SPC). LTM-EEG and MRI were performed in 51 and $85 \%$, respectively, of the patients in the ECPC group vs in 7 and $52 \%$ of the patients in the SPC
\end{abstract}

M. Seeck

margitta.seeck@hcuge.ch

1 EEG and Epilepsy Unit, Division of Neurology, Geneva University Hospitals, 4, Rue Gabrielle-Perret-Gentil, 1205 Geneva, Switzerland

2 Finance Directorate, Geneva University Hospitals, Geneva, Switzerland

3 Medical Direction, Geneva University Hospitals, Geneva, Switzerland

4 Division of Clinical Pharmacology and Toxicology, Geneva University Hospitals, Geneva, Switzerland

5 Division of Emergency Medicine, Geneva University Hospitals, Geneva, Switzerland

6 Division of Neuroradiology, Geneva University Hospitals, Geneva, Switzerland group ( $p<0.001)$. A final diagnosis was obtained in 64 vs $43 \%$ of the patients in the ECPC vs SPC group $(p<0.01)$. Patient attendance at 3-month was $84 \%$ in the ECPC group vs $44 \%$ in the SPC group ( $p<0.001)$. At 12-month follow-up, the delay until the first recurrence was longer in the ECPC group $(p=0.008)$. An early epileptologist-driven protocol is associated with clinical benefit in terms of diagnostic accuracy, follow-up adherence and recurrence. This study highlights the need for epilepsy experts in the early assessment of a first epileptic seizure, starting already in the ED.

Keywords Follow-up $\cdot$ Brain imaging $\cdot$ EEG $\cdot$ Seizure recurrence $\cdot$ Cost analysis

\section{Introduction}

Epilepsy is one of the most frequently occurring neurological diseases, affecting between 0.5 and $1 \%$ of the population [2]. The current definition of epilepsy requires at least one unprovoked seizure together with electroencephalogram (EEG) and brain imaging results in support of an enduring predisposition for subsequent seizures [7, 10].

In most developed countries, people who present with seizures are admitted to the emergency department (ED) and are evaluated by a physician or neurologist but not necessarily by an epilepsy expert. Depending on local resources, the patient is then referred to his general practitioner (GP) or directly to an outpatient clinic. In some countries, patients are encouraged to make an appointment at a "first seizure clinic" managed by epilepsy experts within the next days or weeks. However, in all scenarios, a certain amount of time elapses before the results of further diagnostic tests conducted outside the ED are obtained; this 
delay increases the risk that a patient will not return for follow-up. In our experience, approximately $30 \%$ of all patients do not return for follow-up, although to the best of our knowledge, no study has been conducted.

Several medical organizations [e.g., the Scottish Intercollegiate Guidelines Network (SIGN) [4], the American Academy of Neurology [12, 13], the National Institute for Health and Care Excellence (NICE) in the UK and the German Society of Neurology [6] (GSN)] have established guidelines and provide recommendations on which exams should be performed and when. SIGN [16] and NICE [22] concur that an epilepsy diagnosis should be made by an expert within the 2 weeks following the event and agree that a routine EEG should be conducted as soon as possible. SIGN and GSN postulate that magnetic resonance imaging (MRI) is the exam of choice in patients with epilepsy and on the other hand, NICE recommends sleep electroencephalography over repeated wake EEGs, provided that the first routine recording is negative. Thus, there is no consensus regarding the specific type and timing of advanced diagnostic tests if first-line exams fail to show unequivocal epileptogenic abnormalities.

In the present prospective single-center study, we compared the yield of early comprehensive patient care (ECPC) with standard patient care (SPC) in the setting of a first unprovoked seizure. The ECPC included three consultations by epilepsy experts and all further investigations needed over a short period (see below). We hypothesized the following: (a) ECPC will more often and more rapidly lead to an accurate diagnosis than SPC; (b) ECPC will yield higher adherence to follow-up and treatment monitoring; and (c) Despite higher initial costs related to the comprehensive work-up, ECPC will show long-term cost efficiency due to reduced emergency admissions.

\section{Methods}

\section{Patients}

Between April 2011 and June 2013, we prospectively included all patients admitted to the adult ED (older than 15 years of age) of the University Hospitals of Geneva after a first, presumably unprovoked seizure. Our institution is the only hospital in the canton (province) of Geneva (approx. 400,000 people) that offers continuous neurological care on an emergency basis. Seizures related to withdrawal in chronic drug or alcohol abusers or to metabolic disorders or acute cerebral lesions (less than 7 days) were cataloged as "provoked" and were excluded from the present study. Sleep deprivation and acute alcohol consumption were considered as predisposing factors to unmask subclinical epilepsy disorders but not considered a cause per se. The local ethics board approved the study.

\section{Clinical protocols}

\section{SPC approach}

The SPC consisted of an initial clinical evaluation by an emergency physician who determined the key problem upon admission and requested routine blood tests, electrocardiogram (ECG), and CT. The emergency physician determined the need for a specialized neurology consultation. All patients in the study remained in the ED for observation for between 12 and $24 \mathrm{~h}$. A routine EEG was performed before ED discharge. Based on the patient's history, head-CT and EEG results, a diagnosis and safety advice (driving, swimming, etc.) were formulated and communicated to the patient. The patient was then advised to make an appointment with his GP or with a neurologist of his choice to ensure proper follow-up. A list of neurologists with expertise in epileptology, including those available for in-house consultation, was given to the patient. In the discharge report, MRI and/or long-term EEG (LTM-EEG) were recommended whenever they were deemed necessary for the diagnosis.

\section{ECPC protocol}

This protocol aimed to extend the SPC and to offer expert epilepsy care already in the ED. The patient's history was taken by a board certified epileptologist in the ED and included careful research on the presence of previous seizures or relevant co-morbidities. Depending on the patient's initial evaluation and history, additional tests were arranged. In both the SPC and ECPC groups, a routine EEG was requested before $\mathrm{ED}$ discharge. If $\mathrm{CT}$ and routine EEG were not informative, MRI and LTM-EEG were scheduled within $72 \mathrm{~h}$. If standard EEG findings strongly supported genetic generalized epilepsy (IGE), no imaging was performed. A preliminary diagnosis and socio-professional recommendations (including driving) were communicated.

One to three weeks after the initial event, a follow-up was scheduled in the epilepsy outpatient clinic to communicate the final or most likely diagnosis, the prognosis and the long-term medical and socio-professional consequences. Emergency measures and details about driving limitations were also raised. Whenever a non-epileptic event was suspected, the patient was referred to the appropriate specialist. Finally, a three-month consultation was arranged to assess diagnostic evolution and treatment issues. Between 1 and 2 years after the ED admission, we enquired all the patients (1-year follow-up). 


\section{Study design}

A randomized approach to allocate patients to SPC and ECPC was not possible given the number of intervening physicians and departments involved (Neurology, ED, Radiology). We, therefore, decided upon a two-tiered approach. Patients admitted between April 1, 2011 and March 31, 2012 were included in the SPC group; the SPC protocol has been considered the "established approach" in our institution for many years. Patients admitted between April 1, 2012 and June 6, 2013 were included in the ECPC group.

\section{EEG}

Standard EEGs were recorded for 20 min in agreement with standard recommendations [12, 24], including photic stimulation and hyperventilation. Regarding photic stimulation protocol, stimulation was applied during eyes closed and eyes open. The frequency of stimulation increased from 2 to $60 \mathrm{~Hz}$. During hyperventilation, the patient his asked to breathe deeply during $3 \mathrm{~min}$. Normal routine EEG or non-epileptogenic abnormalities (for instance, focal slowing) were considered inconclusive, requiring further LTM-EEG investigation. LTM-EEG required two additional electrodes over both anterior-temporal regions [18] (for a total of 21 electrodes). The LTM-EEG started in the afternoon up to the next morning for a length of around $18 \mathrm{~h}$. The early awakening period was helpful in identifying genetic generalized epilepsy syndromes [20]. The induction of a generalized spike-wave pattern by photic stimulation or hyperventilation, with or without a history of absence seizures or myoclonic jerks, was considered evidence of genetic generalized epilepsy syndrome. Patients were video-recorded during both routine and LTM-EEG.

\section{Imaging}

Brain CT scans were conducted using a $3 \mathrm{~mm}$ slice thickness. Images were acquired before and after contrast administration. 3T MRI with a 32-channel brain coil was used to obtain high-resolution images. We used a protocol that also enabled us to search for cortical malformations: coronal fast spin echo T2 (FSET2: repetition time (TR) $7520 \mathrm{~ms}$; echo time (TE) $114 \mathrm{~ms}$; voxel size $0.5 \times 0.4 \times$ slice thickness $3 \mathrm{~mm}$ ). This protocol included perpendicular positioning of the slices relative to the hippocampus, 3D Fluid inversion recovery (FLAIR: TR $5000 \mathrm{~ms}$; TE $419 \mathrm{~ms}$; inversion time (TI) $1800 \mathrm{~ms}$; isotropic voxel size $0.9 \times 0.9 \times 0.9 \mathrm{~mm}$ ), diffusion tensor imaging (DTI: TR $8000 \mathrm{~ms}$; TE $84 \mathrm{~ms}$, with 30 gradient directions), and arterial spin labeling (ASL: TR $4000 \mathrm{~ms}$; TE $12 \mathrm{~ms}$, voxel size $3.4 \times 3.4 \times 3.4 \mathrm{~mm}$ with a slice thickness of $4 \mathrm{~mm}$ ). If a tumor was suspected, spin echo (SE) T1 imaging was conducted before and after contrast administration, and a 3D T1 image was acquired after contrast administration. If hemorrhagic lesions were visible on CT, vascular sequences were added. In cases in which autoimmune disorders were a suspected cause of seizure, we added contrast and additional axial gradient echo T2 (GET2: TE $20 \mathrm{~ms}$, TR $832 \mathrm{~ms}$; slice thickness $4 \mathrm{~mm}$ ) or susceptibility weighted imaging (SWI: TE $20 \mathrm{~ms}$; TR $27 \mathrm{~ms} ; 15^{\circ}$ flip angle).

\section{Diagnosis}

The final diagnosis was established as "epileptic seizure" or "cardiovascular", "psychogenic", "other", or "unknown" event (undetermined despite the work-up and expert patient history taking).

An epileptic origin was not based on patient or witness history alone and was retained only if evidence arose from CT, MRI or standard or LTM-EEG. A cardiovascular event diagnosis was given if the patient's history, ECG or blood tests were evocative. As suggested in the literature [3], the diagnosis of both orthostatic hypotension and vasovagal syncope relied mainly on the patient's symptoms and the event history. The diagnosis of psychogenic non-epileptic seizure was based on seizure semiology and its context, negative findings in all other exams and the obligatory presence of a traumatizing event and/or current or past psychiatric disorders. Other final diagnoses were based on the results of further investigations, which were scheduled if deemed necessary. In cases of probable seizure or an unclear diagnosis, we retained a diagnosis of "unknown" event.

\section{Cost analysis}

Cost analysis was performed using the medical catalog utilized in Switzerland (TARMED). We compared hospital costs between both groups as well as the costs associated with additional exams such as brain imaging and electroencephalography studies. All cost results were reported in US\$, using a rounded conversion exchange rate of US $\$ 1=1$ Swiss franc (August 20, 2015).

\section{Statistics}

When we conducted a power analysis, we took into consideration a type I error rate of $5 \%$ (two-sided) and our observation that approximately $30 \%$ of all patients do not complete follow-up consultations once they are discharged from the ED. We therefore calculated that at least 70 patients in each arm were necessary to determine if ECPC leads to a higher attendance rate at epileptology consultations. Group comparisons were performed using Fisher's exact test or the Chi-square test when relative frequencies were considered using SPSS software version 21. 


\section{Results}

\section{Patients}

A total of 183 patients were included in the study, 113 in the ECPC group and 70 in the SPC group. Patients' characteristics are detailed in Table 1.

\section{EEG}

Both groups underwent standard EEG within $72 \mathrm{~h}$ of admission. Among the patients, $73 \%$ underwent the exam within the first $24 \mathrm{~h}$ in the ECPC group vs $66 \%$ in the SPC group $(p=0.17)$. Two patients in each group did not receive a standard EEG. An epileptogenic focus was identified in 13/111 (12\%) of the patients in the ECPC group and in 13/68 (19\%) of the patients in the SPC group $(p=0.17)$. Standard EEG was normal in $66(58 \%)$ patients of the ECPC group and $39(56 \%)$ patients of the SPC group.

LTM-EEG was performed in 33/66 (50\%) of the patients in the ECPC group and in 2/39 (5\%) of the patients in the SPC group due to an unrevealing standard EEG $(p<0.0001)$. In the ECPC group, ten additional cases with epileptogenic anomalies or instances of focal slowing were found, indicating a LTM-EEG yield of $30 \%(10 / 33)$. LTM-EEG revealed subclinical seizure activity in three patients in the ECPC group but in none of the patients in the SPC group (an example is shown in Fig. 1, Table 2).

\section{Brain imaging}

The number of abnormal CT findings did not differ between the groups $(31 / 97$ (32\%) in the ECPC group vs
Table 1 Characteristics of the patients the ECPC (early comprehensive patient care) and SPC (standard patient care) groups

\begin{tabular}{|c|c|c|c|}
\hline & $\mathrm{ECPC}(\%)$ & $\mathrm{SPC}(\%)$ & $p$ value \\
\hline$N$ & 113 & 70 & \\
\hline Age (years) & $43.6 \pm 19.1$ years & $50.1 \pm 22.5$ years & 0.053 \\
\hline \multicolumn{4}{|l|}{ Sex } \\
\hline Female & $51(45)$ & $26(37)$ & \multirow[t]{2}{*}{0.29} \\
\hline Male & $62(55)$ & $44(63)$ & \\
\hline \multicolumn{4}{|l|}{ Personal history } \\
\hline Neurological & $37(33)$ & $26(37)$ & \multirow[t]{5}{*}{0.77} \\
\hline Cardiovascular & $6(5)$ & $5(7)$ & \\
\hline Psychiatric & $11(10)$ & $8(11)$ & \\
\hline Other* & $6(5)$ & $5(7)$ & \\
\hline None & $53(47)$ & $26(37)$ & \\
\hline \multicolumn{4}{|l|}{ Previous seizures } \\
\hline None & $79(70)$ & $61(87)$ & \multirow[t]{4}{*}{0.047} \\
\hline 1 & $11(10)$ & $2(3)$ & \\
\hline$>1$ & $17(15)$ & $4(6)$ & \\
\hline Unknown & $6(5)$ & $3(4)$ & \\
\hline \multicolumn{4}{|l|}{ Timing } \\
\hline Wakefulness & $98(87)$ & $56(80)$ & \multirow[t]{3}{*}{0.0001} \\
\hline Sleep & $14(12)$ & $3(4)$ & \\
\hline Unknown & $1(0.9)$ & $11(16)$ & \\
\hline \multicolumn{4}{|l|}{ Semiology } \\
\hline Focal & $23(20)$ & $21(30)$ & \multirow[t]{3}{*}{0.26} \\
\hline Focal 2nd generalized & $29(26)$ & 13 (19) & \\
\hline Generalized & $61(54)$ & $36(51)$ & \\
\hline \multicolumn{4}{|l|}{ Precipitating factors } \\
\hline Sleep deprivation & $23(20)$ & $8(11)$ & \multirow[t]{5}{*}{0.61} \\
\hline Drugs & $2(18)$ & $2(3)$ & \\
\hline Withdrawal & $7(62)$ & $5(7)$ & \\
\hline Fever/infection & $2(18)$ & $1(1)$ & \\
\hline None & $79(70)$ & $54(77)$ & \\
\hline
\end{tabular}

* All other medical history, e.g., neoplasms, surgical interventions, etc 
A

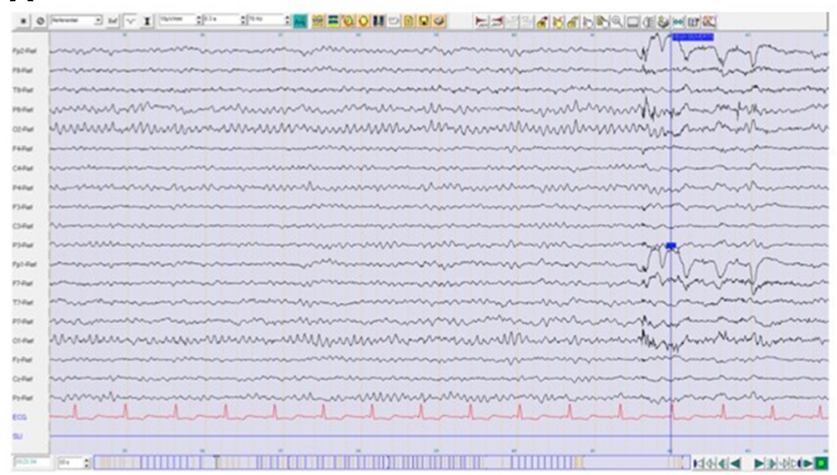

Fig. 1 A 21-year-old patient who experienced a presumably first seizure in the morning in the shower. Review of the patient's history by an epileptologist, and not a general neurologist, identified that this event was already the 4th seizure, all of which occurred in the

Table 2 Standard and long-term EEG performed in both groups

\begin{tabular}{llll}
\hline & ECPC & SPC & $p$ value \\
\hline Standard EEG & & & \\
Abnormal findings & $45 / 111$ & $29 / 68$ & 0.78 \\
Ictal & 3 & 3 & \\
Focal spikes & 5 & 3 & \\
Gen. spikes & 6 & 7 & \\
Focal slowing & 23 & 14 & \\
Gen. slowing & 8 & 2 & \\
LTM-EEG & & & \\
Abnormal findings* & $21 / 50$ & $2 / 7$ & \\
Ictal & 3 & 0 & \\
Focal spikes & 8 & 0 & \\
Gen. spikes & 7 & 1 & \\
Focal slowing & 2 & 1 & \\
Gen. slowing & 1 & 0 &
\end{tabular}

* This table presents all pathological finding in the patient's EEG (e.g., focal spikes were often associated with focal slowing but were categorized as "focal spikes" only). The subcategories of abnormal EEG finding were distributed similarly in both groups: standard EEG $p=0.55$, LTM-EEG $p=0.46$

$27 / 58(47 \%)$ in the SPC group; $p=0.07)$. When CT was inconclusive, MRI was performed in 56/66 (85\%) of the patients in the ECPC group and in 16/31 (52\%) of the patients in the SPC group $(p=0.0005)$. The mean delay to complete the MRI was 12 days in the ECPC group and 32 days in the SPC group $(p=0.001)$. When MRI was performed, no difference in the number of abnormal findings was observed. MRI was abnormal in 17/56 (30\%) of the patients with normal CT in the ECPC group; among these patients, 11 showed epileptogenic lesions. These findings correspond to an additional yield of $20 \%$ (11/56). MRI identified all lesions observed on CT scan (an
B

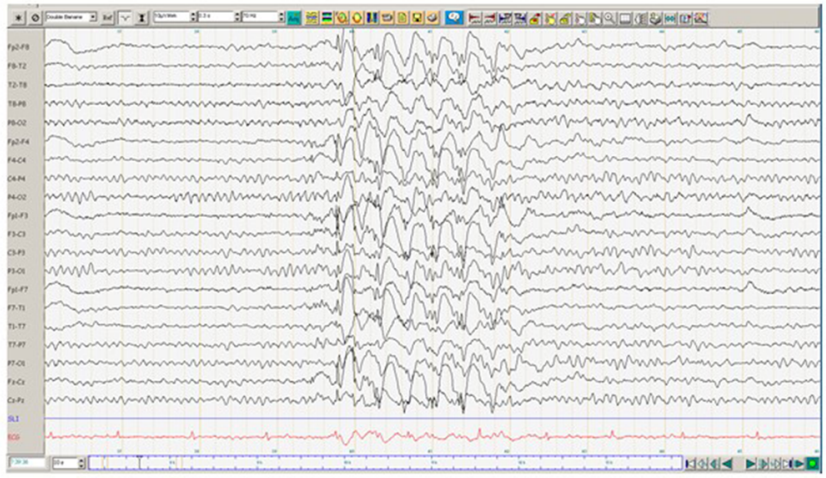

morning. Standard EEG was normal (a), but LTM-EEG showed bursts of generalized polyspike waves, particularly in the morning (b). Genetic generalized epilepsy ("grand mal on awakening") was diagnosed, and lamotrigine successfully introduced

example is shown in Fig. 2). The abnormal MRI findings are detailed in Table 3.

\section{Follow-up}

A follow-up visit was conducted in 95/113 (84\%) of the patients in the ECPC group compared to $31 / 70$ (44\%) of the patients in the SPC group $(p=0.0001)$. The median time to the appointment was 15 days (interquartile ratio (IQR) 10-28) in the ECPC group and 20 days in the SPC group (IQR 6-43). Among the patients diagnosed with epilepsy, those in both groups were equally likely to benefit from antiepileptic drugs (AEDs), which were administered in $42 / 51(82 \%)$ of the patients in the ECPC group and $20 / 24(83 \%)$ of the patients in the SPC group $(p=0.91)$.

The three-month consultation occurred in 63/113 (56\%) of the patients in the ECPC group (with a median delay of 99 days (IQR 69-119) compared to in 18/70 (26\%) of the patients in the SPC group (with a median delay of 129 days (IQR 96-179); $p<0.001$ ). Among those being treated with AEDs, seizure relapses were observed in $8 / 44(18 \%)$ of the patients of the ECPC group and in 1/20 (5\%) of the patients in the SPC group $(p=0.16)$.

At 1 year, 43/113 (38\%) of the patients in the ECPC group were followed by a neurologist vs $14 / 70(20 \%)$ of the patients in the SPC group $(p=0.01)$. Considering only patients with epilepsy, 34/57 (60\%) in the ECPC group were followed by an neurologist vs 7/24 (29\%) in the SPC group $(p=0.01)$. In the ECPC group, $20(18 \%)$ patients were re-admitted to the ED after a new epileptic seizure compared to $7(10 \%)$ patients in the SPC group $(p=0.15)$. The mean time until seizure relapse was 437 days in the ECPC group vs 186 days in the SPC group $(p=0.008)$. 
Fig. 2 A 16-year-old patient with a first generalized tonicclonic seizure. Standard EEG and $\mathrm{CT}$ were unrevealing (a), but MRI showed polymicrogyria in the $\mathrm{R}$ opercular region (b; the right side of the brain is to the left side from the perspective of the viewer; lesion inside the red circle). Focal epilepsy was diagnosed, requiring close monitoring of seizure recurrence, including focal seizures with a more subtle semiology
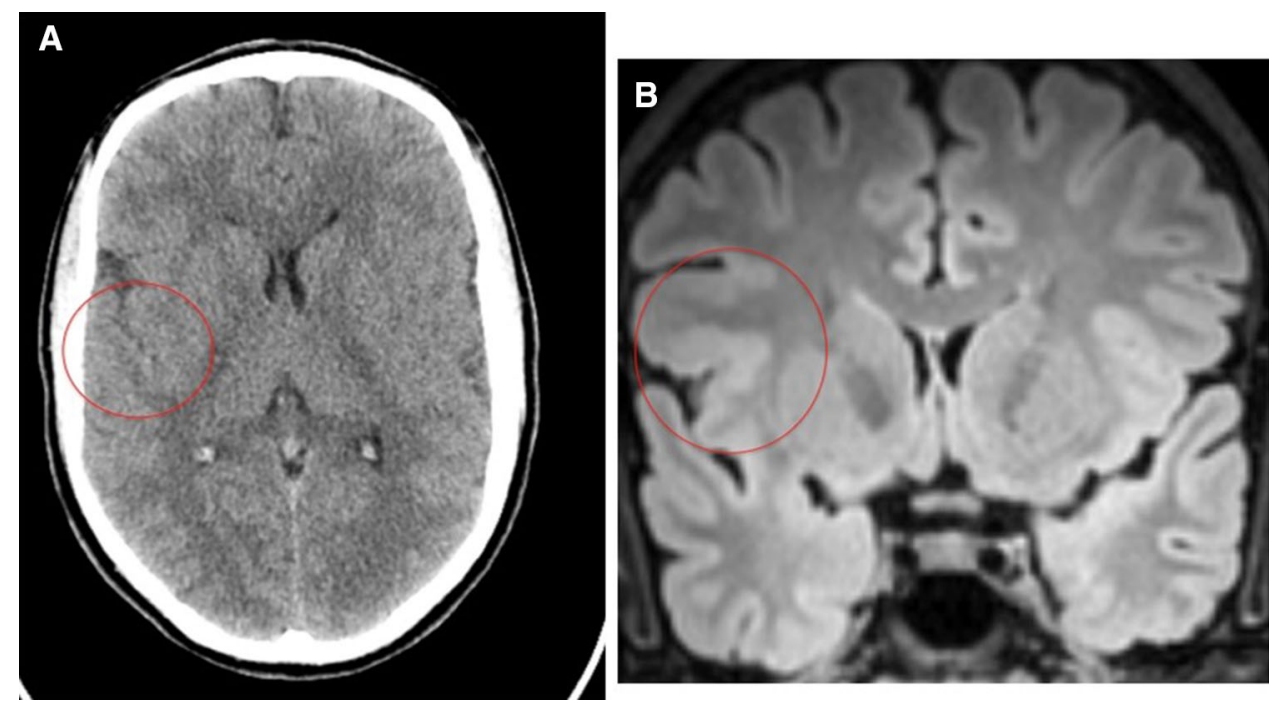

Table 3 Details of MRI abnormalities in both groups

\begin{tabular}{lll}
\hline & ECPC $(\%) N=86$ & SPC $(\%) N=35$ \\
\hline Abnormal findings & $38(44)$ & $16(46)$ \\
Vascular & $7(8)$ & $5(14)$ \\
Tumoral & $15(17)$ & $8(23)$ \\
Metastasis & 3 & 2 \\
Meningioma & 4 & 0 \\
Glioma & 1 & 2 \\
Vascular tumor (cavernoma, hemangioblastoma) & 5 & 2 \\
Cyst & 2 & 2 \\
Trauma & $2(2)$ & 0 \\
Malformation & $9(10)$ & $1(3)$ \\
Hippocampal malformation & 4 & 1 \\
Hippocampal asymmetry/sclerosis & 3 & 0 \\
Temporal cortical dysplasia & 2 & 0 \\
Other & $5(7)$ & $2(6)$ \\
Global or focal atrophy* & 2 & 1 \\
Increased white matter lesions with proximity to the cortex & 2 & 1 \\
Hippocampal hyperintensity & 1 & 0 \\
\hline
\end{tabular}

This table summarizes abnormal MRI findings in 86 patients from the ECPC group and in 35 patients from the SPC group. The pathology categories were similar in both patient groups $(p=0.43)$

* Mammillary body or temporal atrophy

\section{Diagnosis}

At 3 months, a final diagnosis was obtained in 75/113 (66\%) of the patients in the ECPC group compared to 30/70 (43\%) of the patients in the SPC group $(p=0.002)$. Epileptic events were diagnosed in 57/113 (50\%) of the patients in the ECPC group vs 24/70 (34\%) of the patients in the SPC group $(p=0.03)$. A diagnosis of unknown event was significantly less common in the ECPC group than in the SPC group (38/ $113(34 \%)$ in the ECPC group vs 40/70 (57\%) in the SPC group; $p=0.002$ ). All results are detailed in Table 4 .
At 1 year, 21/113 (19\%) of the patients in the ECPC group were diagnosed with an unknown event vs $22 / 70$ $(31 \%)$ of the patients in the SPC group $(p=0.046)$ (Table 5). In both groups, more patients received a final diagnosis at 1 year (ECPC: $p=0.036$, SPC: $p=0.009$ ) because other events enabled correct categorization; however, this was more pronounced for the SPC group. Since diagnoses were based on positive investigations only, no diagnosis changed during follow-up for the already diagnosed patients. On the other hand, a final diagnosis was established for many patients in both groups in 
Table 4 Distribution of diagnoses in the ECPC $(N=113)$ and $\operatorname{SPC}(N=70)$ groups at the 3 -month follow-up

\begin{tabular}{llll}
\hline & ECPC $(\%) N=113$ & SPC $(\%) N=70$ & $p$ value \\
\hline Epileptic seizures & $57(50)$ & $24(34)$ & 0.02 \\
IGE & $10(18)$ & $5(21)$ & \\
Symptomatic & $29(53)$ & $16(64)$ & \\
Cryptogenic & $15(26)$ & $1(4)$ & 0.15 \\
Not classified & $3(5)$ & $2(8)$ & 0.07 \\
Cardiovascular disease & $14(12)$ & $3(8)$ & - \\
Psychogenic & 0 & 0 & 0.54 \\
Other & $4(4)$ & $3(8)$ & 0.003 \\
Unknown & $38(34)$ & $40(57)$ & \\
\hline
\end{tabular}

Table 5 Evolution of diagnostic categorization

\begin{tabular}{|c|c|c|c|c|c|c|c|}
\hline & \multicolumn{2}{|c|}{ Epileptic seizure } & \multicolumn{2}{|c|}{ Non-epileptic seizure } & \multicolumn{2}{|c|}{ Unknown diagnosis } & \multirow[t]{2}{*}{$p$ value } \\
\hline & 3 months & 12 months & 3 months & 12 months & 3 months & 12 months & \\
\hline $\mathrm{ECPC}(N=113)$ & $57(50)$ & $71(63)$ & $18(15)$ & $21(17)$ & $38(34)$ & $21(19)$ & 0.036 \\
\hline SPC $(N=70)$ & $24(34)$ & $38(53)$ & $6(9)$ & $10(10)$ & $40(57)$ & $22(31)$ & 0.009 \\
\hline
\end{tabular}

Follow-up at 12 months enabled a decrease in the number of patients with a diagnosis of "unknown origin" in both the ECPC and SPC groups. Most often, the patients eventually received a diagnosis of "epilepsy". While this was true for the patients in both groups, the evolution of diagnostic categorization was more pronounced in the SPC patients due to the lower initial diagnostic precision the "unknown" category after 1 year of evaluation. This is true for 14 patients in the ECPC and SPC group each, with the diagnostic events being mainly recurrent fits that allowed the identification of the underlying pattern or subsequent positive investigations (e.g., another EEG with postictal abnormalities). Most patients moved from a diagnosis of "unknown" to "epilepsy" (Table 5).

\section{Cost analysis}

Both the costs and resources used indicated a significant difference between the patient cohorts, in line with the number of complementary diagnostic tests performed. In particular, sleep EEG and cranial MRI were more often implemented in the ECPC group than in the SPC group; in contrast, cranial CT was slightly more commonly carried out in the SPC group than in the ECPC group ( 89 vs $83 \%$ ). The ECPC-related procedures were associated with higher costs from pre-hospitalization to outpatient clinic care, i.e., $\$ 1431$ vs $\$ 1035$ as direct medical costs and $\$ 2573$ vs $\$ 2065$ as the global medical management cost. Conversely, no difference was found in the cost of the total number of laboratory tests ( $\$ 383$ vs $\$ 381$ ) or in ED costs.

\section{Discussion}

This study demonstrates the superiority of early comprehensive care by epilepsy experts over the conventional approach in terms of patient follow-up, final diagnosis and seizure recurrence. When the medical history was obtained by epileptologists, critical elements for prognosis were identified compared to when the history was obtained by other professionals in the ED setting. Moreover, when the work-up was organized in the emergency room, within hours after the seizure, adherence to follow-up increased significantly, even 1 year later.

When the follow-up was organized by epilepsy experts, the patients more often and more quickly received MRI and LTM-EEG, which, in turn, increased the diagnostic accuracy of the precipitating event. In fact, our study showed an additional yield of 20 and $30 \%$ for MRI and LTM-EEG, respectively. This additional yield of LTM-EEG, including sleep recording, is comparable to that found in previous studies [15]. The presence of epileptogenic abnormalities after a first seizure is reported in approximately 12-27\% of cases using standard EEG [21] and in up to $58 \%$ when sleep EEG is obtained [11, 14, 23, 25]. The yield of standard EEG is augmented if it is obtained within the first $24 \mathrm{~h}$, e.g., the detection of epileptiform abnormalities increased from 34 to $51 \%$ [11]. In our study, $35 \%$ of patients presented epileptiform abnormalities in their early EEG; these results are similar to those of Prawidal et al. [19] but lower than those reported in the study of King et al. [11], likely because our population included only adults whereas their study included both adults and children. Notably, three patients in the ECPC group but none in the SPC group showed ictal activity on LTM-EEG, which led to immediate treatment initiation and in-house supervision. 
The superiority of MRI over CT is now established knowledge, and this superiority also extends to the ED setting $[2,9,11]$. In a recent study of 764 patients at a "first seizure clinic", $23 \%$ had an epileptogenic lesion on MRI [9], similar to our study. These results suggest that directly ordering an MRI could save time and money if local resources allow rapid in-house MR imaging.

At 12 months, fewer patients in both the ECPC and SPC groups continued to have a diagnosis of "unknown origin". Most of the patients were diagnosed with "epilepsy". Indeed, if MRI and initial EEGs are unrevealing, only the recurrence of events of similar semiology and a subsequent EEG with focal discharges enables a diagnosis of epilepsy. However, if the patient received early comprehensive care, more patients were correctly diagnosed initially. As a result, long-term follow-up was less crucial for obtaining the correct "epilepsy" diagnosis in the ECPC patients than in the SPC patients.

The consequences of structured early follow-up were evident even at the long-term follow-up. Compared to the patients with epilepsy in the SPC group, those in the ECPC group were significantly more likely to be followed by a neurologist 1 year after the index event. The seizure relapse rate did not differ between the two groups, indicating that the type of initial ED care does not influence the disease prognosis itself. However, we showed that the delay between the first event and the next ED re-admission was significantly longer in the ECPC group (437 and 186 days in the ECPC and SPC groups, respectively), which suggests that these patients better understood their condition (i.e., calling the epilepsy expert the next day rather than returning to the ED) and the importance of treatment compliance and lifestyle adjustments (i.e., avoiding precipitating factors) [5]. However, further studies are required to determine if this understanding translates into significant medical and socio-professional longterm effects of ECPC.

Both systematic reviews and cost-of-illness (COI) studies have reported a consistent pattern of markedly higher costs associated with those with uncontrolled or refractory epilepsy [1]. Our cost calculations were consistent with previous COI studies, which estimated that over 1 year (prevalence based), the direct medical costs related to epilepsy mainly involved the costs of return visits and long-term therapy [17]. A total annual cost of $€ 1698$ (\$1919) per patient with active epilepsy treated in private neurological offices was reported in Germany [17], whereas the highest annual cost for this patient group was found in the US, at up to $\$ 13,787$ [8]. Because we are not dealing with chronic epilepsy, the costs of the first seizure work-up are lower. We calculated a difference of approximately $\$ 500$ in favor of SPC. However, our study highlighted that despite the higher upfront investigational cost of ECPC, the patients in this group benefited from fewer subsequent ED and hospital admissions. In the long run, a reduction in admissions reduces health care needs and limits resource consumption. We hypothesize that the direct and indirect economic benefits will become even more apparent beyond the first year due to targeted treatment of conditions mimicking epilepsy (e.g., psychogenic seizures), better drug adherence in patients with epilepsy, earlier curative treatment (surgery), fewer truly intractable patients and, consequently, fewer ED or hospital admissions and less absenteeism at work.

The major limitation of this study is the lack of randomization in assigning patients to SPC or ECPC: we felt that randomization was not practically possible, given the number of implicated teams. In our opinion, successive cohorts in the same ED setting represent the best alternative. Moreover, this is a single-center study, which should be confronted to a multicenter trial to confirm our findings. Another limitation is related to the setting of the study, which was conducted at a university hospital in a country with a rather affluent health care system, which allowed rapid in-house organization of more advanced exams. While this type of care may appear difficult to put into place in smaller hospitals, training of general neurologists, rapid access to MRI, and overnight EEG could also be achieved in non-university settings or in collaboration with university hospitals. The study's setting allowed us to determine the yield of comprehensive patient care by epilepsy experts given that for both protocols, both admission and the initial work-up were completed at the same center (ED). Finally, we did not derive quality-of-life estimates, which could have provided a measure of additional patient-reported outcomes and the benefits of ECPC compared to SPC which represents current ethical standard patient care.

Our data provide new and important information regarding the clinical value of comprehensive patient management after a first epileptic seizure, supporting an early in-hospital approach that is carried out by epilepsy experts. Based on our encouraging results, long-term studies regarding the effects of standard or comprehensive epilepsy care on drug compliance, seizure relapse, quality of life and even mortality should be conducted.

Acknowledgments We thank the EEG and MRI technicians for their efficient and rapid patient care. This work was funded by Swiss National Foundation grants SNSF 140332 (M.S., S.V.), 127608 (F.P., M.S.), 141165 (S.V.) and 146633 (M.S.).

\section{Compliance with ethical standards}

Conflicts of interest The authors declare that they have no conflict of interest.

Ethical standards Our study was approved the ethical committee. 
Financial disclosures L Fisch reports no disclosures, A. Lascano reports no disclosures, N. Vernaz Hegi reports no disclosures, F. Girardin reports no disclosures, V. Kapina reports no disclosures, L. Heydrich reports no disclosures, O. Rutschmann reports no disclosures, F. Sarasin reports no disclosures, M-I. Vargas reports no disclosures, F. Picard reports no disclosures, S. Vulliémoz reports no disclosures, A-C. Héritier-Barras reports no disclosures, and M. Seeck reports consulting and speaker fees from UCB, GSK, and EISAI.

Study funding This work was funded by Swiss National Foundation grants SNSF 140332 (M.S., S.V.), 141165 (S.V.), 146633 and 163398 (M.S.). M.S. received consulting and speaker fees from UCB, GSK and EISAI.

\section{References}

1. Begley CE, Durgin TL (2015) The direct cost of epilepsy in the United States: a systematic review of estimates. Epilepsia 56:1376-1387

2. Berg AT, Shinnar S (1991) The risk of seizure recurrence following a first unprovoked seizure: a quantitative review. Neurology 41:965-972

3. Brignole M, Alboni P, Benditt DG, Bergfeldt L, Blanc JJ, Thomsen PE, Gert van Dijk J, Fitzpatrick A, Hohnloser S, Janousek J, Kapoor W, Kenny RA, Kulakowski P, Masotti G, Moya A, Raviele A, Sutton R, Theodorakis G, Ungar A, Wieling W, Priori SG, Garcia MA, Budaj A, Cowie M, Deckers J, Burgos EF, Lekakis J, Lindhal B, Mazzotta G, Morais J, Oto A, Smiseth O, Menozzi C, Ector H, Vardas P (2004) Guidelines on management (diagnosis and treatment) of syncope-update 2004. Executive summary. Eur Heart J 25:2054-2072

4. Dunn MJ, Breen DP, Davenport RJ, Gray AJ (2005) Early management of adults with an uncomplicated first generalised seizure. Emerg Med J 22:237-242

5. Eatock J, Baker GA (2007) Managing patient adherence and quality of life in epilepsy. Neuropsychiatric Dis Treat 3:117-131

6. Elger (2012) Erster epileptischer Anfall und Epilepsien im Erwachsenenalter. In: Guidelines of the German Neurology Association. http://www.dgn.org/images/red_leitlinien/LL_2012/ pdf/11_01_2012_erster_epileptischer_anfall_und_epilepsien_im_ erwachsenenalter.pdf

7. Fisher RS, van Emde Boas W, Blume W, Elger C, Genton P, Lee P, Engel J Jr (2005) Epileptic seizures and epilepsy: definitions proposed by the International League Against Epilepsy (ILAE) and the International Bureau for Epilepsy (IBE). Epilepsia 46:470-472

8. Gao L, Hu H, Zhao FL, Li SC (2016) Can the direct medical cost of chronic disease be transferred across different countries? Using cost-of-illness studies on type 2 diabetes, epilepsy and schizophrenia as examples. PLoS One 11:e0147169

9. Hakami T, McIntosh A, Todaro M, Lui E, Yerra R, Tan KM, French C, Li S, Desmond P, Matkovic Z, O’Brien TJ (2013) MRI-identified pathology in adults with new-onset seizures. Neurology 81:920-927

10. Hauser WA, Annegers JF, Kurland LT (1993) Incidence of epilepsy and unprovoked seizures in Rochester, Minnesota: 1935-1984. Epilepsia 34:453-468
11. King MA, Newton MR, Jackson GD, Fitt GJ, Mitchell LA, Silvapulle MJ, Berkovic SF (1998) Epileptology of the first-seizure presentation: a clinical, electroencephalographic, and magnetic resonance imaging study of 300 consecutive patients. Lancet 352:1007-1011

12. Krumholz A, Wiebe S, Gronseth G, Shinnar S, Levisohn P, Ting T, Hopp J, Shafer P, Morris H, Seiden L, Barkley G, French J (2007) Practice parameter: evaluating an apparent unprovoked first seizure in adults (an evidence-based review): report of the Quality Standards Subcommittee of the American Academy of Neurology and the American Epilepsy Society. Neurology 69:1996-2007

13. Krumholz A, Wiebe S, Gronseth GS, Gloss DS, Sanchez AM, Kabir AA, Liferidge AT, Martello JP, Kanner AM, Shinnar S, Hopp JL, French JA (2015) Evidence-Based Guideline: management of an Unprovoked First Seizure in Adults: Report of the Guideline Development Subcommittee of the American Academy of Neurology and the American Epilepsy Society. Epilepsy Curr/Am Epilepsy Soc 15:144-152

14. Mendez M, Radtke RA (2001) Interactions between sleep and epilepsy. J Clin Neurophysiol 18:106-127

15. Michel V, Mazzola L, Lemesle M, Vercueil L (2015) Long-term EEG in adults: sleep-deprived EEG (SDE), ambulatory EEG (Amb-EEG) and long-term video-EEG recording (LTVER). Clin Neurophysiol 45:47-64

16. Network SIGN (2001) Healthcare Improvement Scotland. http:// sign.ac.uk/guidelines/fulltext/143/index.html

17. Noda AH, Hermsen A, Berkenfeld R, Dennig D, Endrass G, Kaltofen J, Safavi A, Wiehler S, Carl G, Meier U, Elger CE, Menzler K, Knake S, Rosenow F, Strzelczyk A (2015) Evaluation of costs of epilepsy using an electronic practice management software in Germany. Seizure 26:49-55

18. Nowack WJ, Janati A, Metzer WS, Nickols J (1988) The anterior temporal electrode in the EEG of the adult. Clin Electroencephalogr 19:199-204

19. Paliwal P, Wakerley BR, Yeo LL, Ali KM, Ibrahim I, WilderSmith E, Sim TB, Pohlmann-Eden B, Rathakrishnan R (2015) Early electroencephalography in patients with Emergency Room diagnoses of suspected new-onset seizures: diagnostic yield and impact on clinical decision-making. Seizure 31:22-26

20. Panayiotopoulos CP (2005) Idiopathic generalized epilepsy. In: The Epilepsies: Seizures, Syndromes and Management. Bladon Medical Publishing, Oxfordshire, UK

21. Pohlmann-Eden B, Newton M (2008) First seizure: EEG and neuroimaging following an epileptic seizure. Epilepsia 49(Suppl 1):19-25

22. Pugh MJ, Leykum LK, Lanham HJ, Finley EP, Noel PH, McMillan KK, Pugh JA (2014) Implementation of the Epilepsy Center of Excellence to improve access to and quality of careprotocol for a mixed methods study. Implement Sci 9:44

23. Schreiner A, Pohlmann-Eden B (2003) Value of the early electroencephalogram after a first unprovoked seizure. Clin Electroencephalogr 34:140-144

24. Smith SJ (2005) EEG in the diagnosis, classification, and management of patients with epilepsy. J Neurol Neurosurg Psychiatry 76(Suppl 2):ii2-ii7

25. van Donselaar CA, Schimsheimer RJ, Geerts AT, Declerck AC (1992) Value of the electroencephalogram in adult patients with untreated idiopathic first seizures. Arch Neurol 49:231-237 